Original article:

\title{
Comparing Diagnostic Value of Renal Parenchymal Resistive Index And Cortical Echogenicity in Chronic Kidney Disease Patients \\ Dria Anggraeny Sutikno ${ }^{1}$, Nurdopo Baskoro ${ }^{2}$
}

\begin{abstract}
:
Background: Chronic kidney disease (CKD) is a clinically impaired kidney degradation syndrome, which commonly is diagnosed based on glomerulus filtration rate (GFR). Renal parenchymal resistive index and the renal cortex echogenicity are ultrasound parameters that have been reported correlate with GFR values. This study aims to determine the sensitivity, specificity, positive predictive value, and negative predictive value between renal intra-parenchymal resistive index and renal cortical echogenicitybased on GFR in CKD patients. Materials and Methods: This study is a cross sectional design. A renal ultrasound examination was performed to forty one CKD patients to assess the resistive index of the renal intra-parenchymal artery and the echogenicityof the renal cortex. The creatinine serum levels were obtained from the patients, as the gold standard of CKD diagnosis. Statistical data processing uses diagnostic test and Inter class Correlation Coefficients (ICC). Results: The sensitivity, specificity, positive predictive value, and negative predictive value of renal intraparenchymal resistive indexes were $23 \%, 79 \%$, $33 \%$, and $69 \%$ respectively. Sensitivity, specificity, positive predictive value, and negative predictive value between renal cortex echogenicitywere $23 \%, 96 \%, 75 \%$, and $73 \%$ respectively. The ICC analysisreported a Single Rater value of 0.1538 and Average of Raters 0.3528. Conclusion: Renal intra-parenchymal resistive artery was more specific than renal cortex echogenicity for diagnosing patients with chronic kidney disease.
\end{abstract}

Kevwords: Resistive Index, renal cortex echogenicity, chronic kidney disease, glomerulus filtration rate (GFR).

International Journal of Human and Health Sciences Vol. 04 No. 03 July'20 Page : 194-199 DOI: http://dx.doi.org/10.31344/ijhhs.v4i3.200

\section{Background}

Chronic kidney disease (CKD), also known as endstage renal disease, is a syndrome characterized by progressive and irreversible kidney loss, and the incidence of chronic kidney disease nowadays is increasing rapidly. The incidence of chronic kidney disease significantly increases annually. The increasing number of patients with chronic kidney disease also leads to an increase in the number of patients undergoing hemodialysis. Based on a survey conducted by Association of Nephrology Indonesia in 2009-2012, it was reported that there was an increase in the percentage of new CKD patients by $50 \%$ in Indonesia. ${ }^{1}$ The prevalence of chronic kidney disease increases along with the increasing number of elderly population and the incidence of diabetes mellitus and hypertension. About 1 of 10 global populations experiences chronic kidney disease at a particular stage. The global prevalence of chronic kidney disease of $13.4 \%$ and was reported as the 27 th leading cause of death in the world in 1990 and increased to 18th in $2010 .^{1,2}$ In Indonesia, renal disease treatment is the second largest financing ranking of national

1. Dria Anggraeny Sutikno, Department of Radiology, Faculty of Medicine, Universitas Islam Sultan Agung

2. Nurdopo Baskoro, Department of Radiology, Faculty of Medicine, Diponegoro University / dr, Kariadi Hospital

Correspondence to: Dria Anggraeny Sutikno, MD. Radiologist, Department of Radiology; Universitas Islam Sultan Agung Jl. Kaligawe KM 4 Semarang Indonesia 50112.

Phone number :+6281326707161, Email : dria.anggraeny@unissula.ac.id 
International Journal of Human and Health Sciences Vol. 04 No. 03 July'20

health insurance, it is ranked after heart disease. According to the data obtained from PT Askes (one of Indonesian health insurance), there were approximately 14.3 million people with late stage renal failure currently undergoing treatment with a prevalence of 433 per population. This number is expected to increase to over 200 million by $2025^{1,2}$ Clinically, the basic diagnosis for chronic kidney disease is to assess the glomerulus filtration rate. Glomerular filtration rates and renal tubules have a strong contribution in maintaining renal function known as glomerulus tubular balance and negative glomerulus tubular feedback. Glomerulus and renal tubules play a vital role in maintaining fluid balance.Any condition that causes an increase in fluid and $\mathrm{Na}$ in the macula densa (distal tubule) will eventually trigger a feedback mechanism that will cause a decrease in the filtration rate of the nephron. ${ }^{3}$ The classification of chronic kidney disease based on glomerulus filtration rate (GFR) was reported on table 1.

Table 1 Classification of chronic kidney disease
based on GFR.(4)

In the field of nephrology, diagnostic techniques would include various aspects such as clinical history, physical examination, laboratory tests, scintigraphy, diagnostic imaging techniques and renal biopsy. In the case of nephropathy, ultrasonography is a first-line imaging technique, which; through careful sonographic scanning, it can be a helpfulinstrument to distinguish acute and chronic renal failure. ${ }^{4}$ In addition, ultrasound can also be used to follow up the treatment of the disease, to guide the biopsyneedle and others. Ultrasound techniques can visualize renal pelvic conditions, assess renal dimensions and parenchymal echogenicity, visualize Doppler color-power signals and measure parenchymal resistive index. Taken together, this data can provide useful clues to the diagnosis and help to reduce the possible differential diagnoses. ${ }^{5}$

A positive correlation between the echogenicity of therenal cortex and interstitial changes was reported in 25 patients, but it was found no association between these changes and glomerular lesions. ${ }^{5}$ However, Hricak et al. in 1982, found a positive correlation between echogenicity increase of the renal cortex and glomerular changes. ${ }^{7}$ Meanwhile, according to a study conducted by Siddappa et al in 2013, it was evident that the echogenicity of the renal cortex was one of the parameter that had significant correlation with serum creatinine levels compared to other sonographic parameters such as renal axis length, parenchymal thickness, and cortical thickness. ${ }^{7}$ Echogenicity grading of the renal cortex is shown in Table 2.

Table 2. Classification of renal parenchymal echogenicityaccording to Brenbridge et al. ${ }^{8}$

\begin{tabular}{ll}
\hline Grade 1 & Echogenicity is less than liver/spleen \\
\hline Grade 2 & Echogenicity is similar to liver/spleen \\
\hline Grade 3 & Echogenicity are more than liver/spleen \\
\hline Grade 4 & Echogenicity equals central renal sinus or portal vein \\
\hline
\end{tabular}

Examination of renal intra-parenchymal artery resistive index happened to have a correlation with estimated renal glomerular filtration rate in hypertensive disease. In another study, it was also mentioned that renal Doppler ultrasound examination had an important role in the prognosis in patients with chronic kidney disease. In chronic kidney disease, vascular compliance might occur and increased peripheral resistance, which led to a decrease in blood flow to the kidney and glomerular filtration rate. Doppler ultrasound examination, especially resistive index could provide useful information related to the above disorder. ${ }^{9}$

The normal resistivescore of the renal index is about 0.60 to 0.65 which is maintained constantly as long as the structure of the parenchyma and renal function remains normal. In neonate patients, the resistive index appears to be higher and gradually decreases by increasing of age and will be persists at 8-10 years of age. This score is similar to the resistive index of normal adult. However, in the elderly ( $>50$ years) the resistive index appears elevated, associated with changes in vascular structures. Therefore, normal resistive index score ranges from 0.6 to $0.7 .{ }^{9}$ 


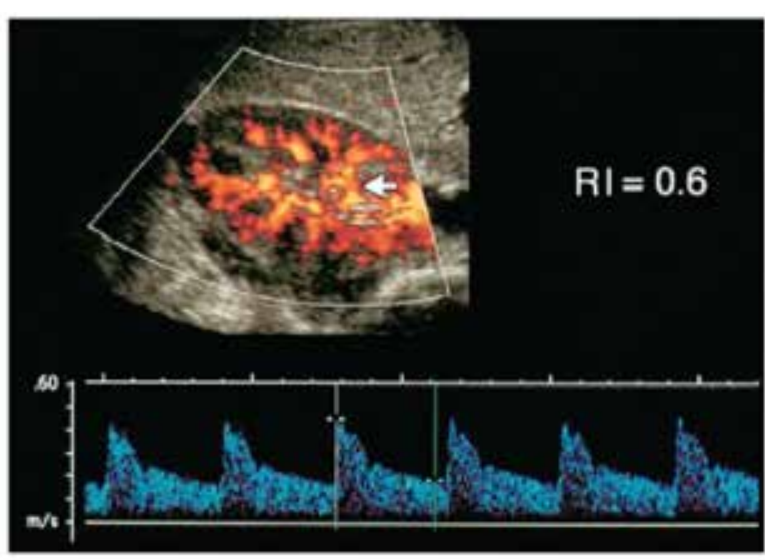

Figure 1. Resistive index is normal in healthy female patients, 25 years of age. Color Doppler sonogram is used to identify interlobar artery (arrow). ${ }^{9}$

Based on the description above, resistive index examination and echogenicity of renal cortex are ultrasound parameters, which could be related to glomerulus filtration rate. However, to the best of our knowledge, there has been no publication that focused on which parameter that contributes the most on the depletion of glomerulus filtration rate in patients with chronic kidney disease. Considering that, the authors would like to examine sensitivity, specificity, positive predictive value, and negative predictive value of renal intra-parenchymal artery resistive index andechogenicity of the renal cortex based on renal glomerular filtration rate as gold standard diagnosis in patients with chronic kidney disease.

\section{Research Methods}

This study was a cross sectional study with a diagnostic test conducted at Dr. KariadiGeneral Hospital from August to October 2017. The study sampleswere patients with chronic kidney disease with inclusion criteria: GFR $\leq 90 \mathrm{~mL} / \mathrm{min} / 1.73 \mathrm{~m} 2$ (based on GFR MDRD formula), age $\geq 19$ years, and underwent ultrasound examination at $\mathrm{dr}$. Kariadi General Hospital Radiology Diagnostic Department, and agreed to be the subject of thestudy. Patients with nephrolithiasis, renal anatomic abnormalities, hydro-nephrosis, renal malignancies, undergoing kidney replacement therapy (hemodialysis, peritoneal dialysis, and renal transplant), fatty liver, and ascites were excluded from the sample. The total samples was 41 patients, and were collected using consecutive sampling method, which was selected based on their arrival to the Department of Radiology Diagnostic Dr. Kariadi, Semarang to undergo renal ultrasound (abdominal). The result of creatinine serum examination was obtained from the hospital laboratory test and the result of the test was attached to the medical record of the patients. CKD patients who agreed to take part the study and had signed a written informed consent agreement with GFR $\leq 90 \mathrm{~mL} / \mathrm{min} / 1.73 \mathrm{~m} 2$ were underwent renal ultrasound. The ultrasound was performed using the curve probe with B-mode ultrasound and Doppler ultrasound to obtain a resistive index of the renal intra-parenchymal artery in the upper, mid and lower poles. The sample volume was set as small as possible $(1 \mathrm{~mm})$ into the inter-lobar artery with a narrow angle. The final result of the resistive index value was the average of the three measurements mentioned above. However, if it was not possible to obtain a renal intra-parenchymal artery in all three poles, then only one intraparenchymal artery could be visualized. There was also an examination of the echogenicity of the renal cortex by using B-mode ultrasound. One person counted as one sample, where ultrasound examination was performed on both kidneys in each sample. Resistive index scores and the degree of parenchymal echogenicitywere taken from one of the kidneys that had the highest scores.

Processing Techniques and Data Analysis.

Data were expressed in 2 x 2tables, and then diagnostic values of resistive index of renal intraparenchymal artery and the echogenicity of the renal cortex were calculated. Furthermore, Kappa conformity testing was performed between the renal intra-parenchymal resistive index and renal cortical echogenicity index to GFR.

\section{Results}

The prevalence of female CKD patients (63.4\%) was higher than that of the male CKD patients $(36.6 \%)$ and the most prevalent agewas 41-60 years $(56.1 \%)$.

Table 3. General characteristics of subjects

\begin{tabular}{c|c|c|c}
\hline & Characteristics & Sample & Percentage (\%) \\
\hline \multirow{3}{*}{ Age } & $21-40$ & 6 & 14.6 \\
& $41-60$ & 23 & 56.1 \\
\multirow{4}{*}{ Gender } & $>60$ years & 12 & 29.3 \\
& Male & 15 & 36.6 \\
GFR & Female & 26 & 63.4 \\
& GFR $\geq 15 \mathrm{ml} / \mathrm{min}$ & 13 & 31.7 \\
Echogenicity & GFR $<15 \mathrm{ml} / \mathrm{min}$ & 28 & 68.3 \\
& Grade 1 & 4 & 9.8 \\
Resistive & Grade 2-4 & 37 & 90.2 \\
Index (RI) & $0.60-0.70$ & 9 & 22.0 \\
& $>0.70$ & 32 & 78.0
\end{tabular}

There were 13 patients categorized as normal to severe kidney function depletion, with GFR score $\geq 15 \mathrm{ml} / \mathrm{min}$, and there were 28 patients 
suffered from very severe kidney function depletion, with the GFR score $<15 \mathrm{ml} / \mathrm{min}$. Based on the echogenicity of the renal cortex obtained by B-mode ultrasound examination, 4 patients were considered as normal with homogeneity grade 1 and there were 37 patients withhomogeneity grade 2-4. Meanwhile, based on the resistive index (RI) of renal intra-parenchymal artery obtained by Doppler ultrasound examination, there were 9 patients with normal RI scores, 0.60 to 0.70 and there were 32 patients had RI scores more than 0.70 .

The analysis indicated that the sensitivity, specificity, positive predictive value, and negative predictive value of renal cortex echogenicity were $23 \%, 96 \%, 75 \%$, and $73 \%$ respectively and the sensitivity, specificity, positive predictive value, and negative predictive value of renal intraparenchymal resistive index were $23 \%, 79 \%$, $33 \%$, and $69 \%$ respectively.

Table 4. Diagnostic test of renal cortex echogenicity on the GFR value

\begin{tabular}{llll}
\hline \multirow{2}{*}{ Ekogenisitas } & GFR & & \multirow{2}{*}{ Total } \\
\cline { 2 - 3 } & $\begin{array}{l}\mathbf{N} \\
(\geq 15 \mathrm{ml} / \mathrm{min})\end{array}$ & $\begin{array}{l}\text { TN } \\
(<15 \mathrm{ml} / \mathrm{min})\end{array}$ \\
\hline $\mathbf{N}$ & 3 & 1 & 4 \\
$($ Grade 1$)$ & $23.1 \%$ & $3.6 \%$ & $9.8 \%$ \\
TN & 10 & 27 & 37 \\
(Grade $2-4)$ & $76.9 \%$ & $96.4 \%$ & $90.2 \%$ \\
\hline \multirow{2}{*}{ Total } & 13 & 28 & 41 \\
& $100.0 \%$ & $100.0 \%$ & $100.0 \%$ \\
\hline
\end{tabular}

Table 5. Diagnostic test of renal cortex echogenicity on the GFR value

\begin{tabular}{llll}
\hline \multirow{2}{*}{ Resistive Index } & GFR & \multicolumn{2}{c}{ Total } \\
\cline { 2 - 4 } & $\mathbf{N}$ & $\mathbf{T N}$ & \\
\hline $\mathbf{N}$ & 3 & 6 & $\mathrm{~m} / \mathrm{min})$ \\
$(<15 \mathrm{ml} / \mathrm{min})$ & \\
$\mathbf{( 0 . 6 0 - 0 . 7 0 )}$ & $23.1 \%$ & $21.4 \%$ & $22.0 \%$ \\
$\mathbf{T N}$ & 10 & 22 & 32 \\
$(>\mathbf{0 . 7 0 )}$ & $76.9 \%$ & $78.6 \%$ & $78.0 \%$ \\
\hline \multirow{2}{*}{ Total } & 13 & 28 & 41 \\
\hline & $100.0 \%$ & $100.0 \%$ & $100.0 \%$ \\
\hline
\end{tabular}

The results of kappa testindicated that the kappa coefficient of the echogenicity of the renal cortex to GFR was $0.239(\mathrm{p}=0.05)$ whereas the coefficient of kappa of resistive index of renal intra-parenchymal artery to GFR was 0.018 ( $\mathrm{p}=$ 0.91).The ICC indicated poor agreement among the variables.

\section{Discussion}

Based on the echogenicity of the renal cortex, it was indicated that CKD patients with echogenicity grade 2-4were higher in number compared to those whose normal echogenicity. This result was in accordance with previous study which reported a linear correlation between sonographic grading in renal parenchyma with GFR scores. The stud reported that CKD patients with grade 2-4 cortical echogenicity were four times as much as patients with the grade lechogenicity. ${ }^{10} \mathrm{O}$ ther literature reported a positive correlation between the increase of renal cortex echogenicity and glomerular changes. The increase of echogenicity of the renal cortex could be due to changes in perfusion, cell infiltration and deposition of connective tissue, calcium, and fat. ${ }^{5}$ In addition, there was further nephron damage in CKD patients that led to tubule-interstitial fibrosis. ${ }^{7}$

This study reported that CKD patients with a resistive index more than 0.70 were higher in number compared to CKD patients with normal resistive index. Previous stud also reported that $77 \%$ patients with high score of GFR would have high resistive index $(>0.70) .{ }^{10}$ This can be understood as CKD patients might suffer from nephron damage and tubule-interstitial fibrosis, which associated with renal intra-parenchymal fibrosis. Those situations might lead to resistive index increase. ${ }^{9}$

The finding indicated that echogenicity of the renal cortex had a more significant diagnostic value in CKD patients based on GFR than the renal intra-parenchymal resistive index. It wasalso reportedpreviously that the echogenicity of the renal cortex had a good correlation with the severity of interstitial changes in biopsy. ${ }^{11} \mathrm{Focal}$ interstitial changes tend to cause minimal increase in the echogenicity of the cortex and diffuse cicatrix tissue leading to wider changes in the echogenicity of the renal cortex. It was also reported that renal echogenicity has a strong correlation to histologic parameters, such as glomerular sclerosis, tubular atrophy, interstitial fibrosis, and interstitial inflammation. Renal cortical echogenicity had statistically significant positive correlation with the severity of global sclerosis, focal tubular atrophy, and the number of hyaline cylinders in the glomerulus and focal leukocyte infiltration. ${ }^{11}$ On the other hand, however, it was reported previously that there was similarities of resistive index in patients with normal and moderately thickened intima layers. However, the resistive index was reported tend to increase in patients with severe arteriosclerosis. There was no correlation between resistive index and glomerulosclerosis as well. ${ }^{10}$ This study concluded that the resistive index was less sensitive to histologic changes of 
renal parenchyma when compared to renal cortex echogenicity.

A study conducted by Yaprak et $\mathrm{a}^{12}$ reported that the renal cortex echogenicity had the strongest sonography parameter correlation against e-GFR than the length of the kidney and the thickness of cortex. In addition, in renal biopsy studies, it was shown that the renal cortex echogenicity was also associated with glomerulosclerosis, tubular atrophy, and interstitial fibrosis leading to irreversible kidney function disorder.Moreover, based on a study by Tublin et $\mathrm{al}^{9}$ it was known that the resistive index relied on volume (compliance) and blood vessel resistance, and became less dependent on blood vessel resistance when compliance was reduced, and became increasingly independent when volume was equal to zero. The study also reported that the resistive index alone, without considering other factors, could not be used to determine the differential diagnosis of intrinsic renal disease due to the complex histologic effects on compliance and blood vessel resistance. ${ }^{9}$ Thus, in the absence of other abnormalities affecting resistance renal intra-parenchymal blood vessels, resistive index might give false negative results. Considering that, the authors suggested that the echogenicity of the renal cortex was more representative of the histologic changes of the renal parenchyma in CKD patients. These factors also led to a poor agreement among the three research variables.

\section{Conclusions And Suggestions}

The study indicated that renal intra-parenchymal resistive artery was more specific than renal cortex echogenicity for diagnosing patients with chronic kidney disease. This may be due to the echogenicity of the renal cortex being more likely to visualize the histologic condition of the kidneys than the resistive index, which depends not only on renal intra-parenchymal vascular conditions. The absence of other abnormalities affecting intraparenchymal renal resistance resistive index may give false negative results.

For further study, the authors suggest that resistive index examination could be performed by using linear probes to obtain accurate data of arcuate artery or renal inter-lobar artery. To eliminate the effect of subjectivity in the assessment of the echogenicity of renal parenchyma, further study could be done with more than one assessor. In addition, a longer duration of researchwas needed for obtaining accurate resistive index examination.

\section{Acknowledgement}

The author would like to thank the head of Department of Radiology, dr. Kariadi Hospital and research associates in the center for their assistance in conducting the study. The authors also wish to thank Faculty of Medicine Universitas Islam Sultan Agung, for funding the project. They also would like to thank Endang Lestari for language editing, Darminto MD for his assistance in the statistical analysis and Putri Rohimah Ayuningtyas for her assistance in submitting the manuscript.

\section{Conflict of Interest}

The authors declare that they have no competing interests. The authors alone are responsible for the writing and content of this paper.

\section{Ethical approval and consent to participate}

The study was approved by the Bioethics Committee for Medical/ Health Research Faculty of Medicine, Diponegoro University and dr. Kariadi Hospital (Letter No. 573/EC/FK-RSDK/ IX2017) and was conducted at the Department of Radiology dr. Kariadi Hospital. Participants would not pose to physical risk for taking part this study. The respondents were informed that their participation in this study was on a voluntary basis. Consent was implied by the participants' agreements to take part the study and their signature on written informed consent agreement. To ensure confidentiality we anonymized the data.

\section{Source of Funding}

This project was funded by Faculty of Medicine

Universitas Islam Sultan Agung, under the scheme of Internal Research Funding.

\section{Authors Contribution:}

Study design: Dria A. Sutikno, Nurdopo Baskoro Data gathering: Dria A. Sutikno, Nurdopo Baskoro Writing and submitting manuscript: Dria A. Sutikno, Nurdopo Baskoro

Editing and approval of final draft: Dria A.

Sutikno, Nurdopo Baskoro

\section{Consent for publication}

Not applicable 
International Journal of Human and Health Sciences Vol. 04 No. 03 July'20

\section{References:}

1. Prodjosudjadi WSA. End Stage Renal Disease in Indonesia: Treatment Development. Spring. 2009;19:33-6.

2. Prodjosudjadi WSA Incidence, Prevalence, Treatment and Cost of End-Stage Renal Disease in Indonesia. Ethn Dis. 2006;16:14-6.

3. Sandilands EA, Dhaun N, Dear JW, Webb DJ. Measurement of renal function in patients with chronic kidney disease. $\mathrm{Br} \mathrm{J}$ Clin Pharmacol. 2013;76(4):504-15.

4. Nainggolan T. The Correlation Between Cortical Thickness And Renal Lenght And Estimated Glomerular Filtration Rate (eGFR) In Chronic Kidney Disease. Vol. 1. Makassar: Universitas Hasanuddin, 2014.

5. Fiorini F, Barozzi L. The role of ultrasonography in the study of medical nephropathy. J Ultrasound. 2007;10(4):161-7.

6. Hricak H, Cruz C, Romanski R, Uniewski M., Levin NW, Madrazo BL, et al. Parenchymal Disease. Radiology. 1982;144:141-7.

7. Siddappa JK, Singla S, Al Ameen M, Rakshith SC, Kumar N. Correlation of Ultrasnographic Parameters with Serum Creatinin in Chronic Kidney Disease. J
Clin Imaging Sci. 2013;3(28):1-6.

8. Brenbridge AN, Chevalier RL, Kaiser DL. Increased Renal Cortical Echogenicity i n Pediatric Renal Disease : Histopathologic Correlations. J Clin Ultrasound. 1986;14(October):595-600.

9. Tublin ME, Bude RO, Platt JF. The resistive index in renal Doppler sonography: Where do we stand? Am J Roentgenol. 2003;180(4):885-92.

10. Shivashankara VU, Shivalli S, Santhosh Pai BH, Acharya KD, Gopalakrishnan R, Srikanth V, et al. A comparative study of sonographic grading of renal parenchymal changes and estimated glomerular filtration rate (EGFR) using modified diet in renal disease formula. J Clin Diagnostic Res. 2016;10(2):TC09-TC11.

11. Singh A, Gupta K, Chander R, Vira M. Sonographic Grading of Renal Cortical Echogenicity and Raised Serum Creatinine in Patients With Chronic Kidney Disease. J Evol Med Dent Sci [Internet]. 2016;5(38):2279-86.

12. Yaprak M, Çakır Ö, Nuri M, Ramazan T. Role of ultrasonographic chronic kidney disease score in the assessment of chronic kidney disease. Int Urol Nephrol. 2017;49:123-31. 